\title{
Etude épidémiologique et clinique sur les encéphalites aigües chez les enfants âgés de 0 à 15 ans dans le Département de pédiatrie CHU Gabriel Toure.
}

\section{Epidemiological and clinical study on acute encephalitis in children aged 0-15 years in the pediatric department CHU Gabriel Toure.}

\author{
Coulibaly $\mathrm{O}^{1}$, Dicko Traore $\mathrm{F}^{1}$, Doumbia $\mathrm{AK}^{1}$, Diall $\mathrm{HG}^{1}$, Diakité $\mathrm{AA}^{1}$, Dembele $\mathrm{A}^{1}$, Togo $\mathrm{P}^{1}$, Traore $\mathrm{F}^{1}$, Maîga
}

$\mathrm{B}^{1}$, Sacko $\mathrm{K}^{1}$, Konaté $\mathrm{D}^{1}$, Cissé $\mathrm{ME}^{1}$, Touré $\mathrm{A}^{1}$, Traore $\mathrm{I}^{1}$, Koné $\mathrm{I}^{1}$, Ahamadou $\mathrm{I}^{1}$ Kané $\mathrm{B}^{2}$, Sylla $\mathrm{M}^{1}$, Togo $^{1}$,

Sidibé $\mathrm{T}^{1}$.

1. Centre Hospitalo-Universitaire Gabriel Touré

2. Hôpital du Mali

Auteur correspondant : Dr Oumar COULIBALY, Département de pédiatrie, CHU Gabriel Toure, Bamako,

Email : cheickcoul1@live.fr

\section{Résumé}

Introduction: Les encéphalites aigües sont une cause majeure de morbidité et de mortalité en pédiatrie ; le but de notre étude était d'étudier les aspects épidémiologiques et cliniques des encéphalites aigües chez les enfants âgés de 0 à 15 ans dans le département de pédiatrie du CHU Gabriel Toure. Méthodologie : Il s'est agi d'une étude descriptive sur 12 mois du $1^{\text {er }}$ Janvier au 31 Décembre 2015 au département de pédiatrie du CHU Gabriel Toure Bamako Mali. Tous les dossiers présentant une encéphalite aigüe ont été inclus. Résultats : Ainsi 34 dossiers d'enfants présentant une encéphalite aigüe sur un total de 4727 hospitalisés en pédiatrie ont été analysés soit une fréquence de $0,72 \%$. Environ Quarante sept pour cent $(47,1 \%)$ des enfants avaient un âge compris entre 12 et 59 mois. Le sex- ratio était de 1,8 en faveur des garçons; la fièvre plus la convulsion étaient présente dans $70,6 \%$, suivie du coma $41,2 \%$. Le taux de mortalité était $6 \%$, et $64,7 \%$ ont gardés des séquelles neurologiques.

Conclusion: Les encéphalites aigües constituent une cause importante de séquelles neurologiques chez l'enfant à cause des difficultés diagnostiques et de prise en charge. Mots clés : Encéphalites aigües, Santé publique, Enfant, Séquelles.

\section{Abstract}

Introduction: Acute encephalitis is a major cause of morbidity and mortality in pediatrics; The aim of our study was to study the epidemiological and clinical aspects of acute encephalitis in children aged 0-15 years in the pediatric department of CHU Gabriel Toure. Methodology: This was a 12-month descriptive study from January 1 st to December 31st, 2015 in the pediatric department of the Gabriel Toure Bamako Mali Teaching Hospital. All cases with acute encephalitis were included.Results: Thus 34 cases of children with acute encephalitis out of a total of 4,727 hospitalized pediatric patients were analyzed, a frequency of $0.72 \%$. Approximately Forty-seven percent $(47.1 \%)$ of the children were between 12 and 59 months old. The sex ratio was 1.8 in favor of boys; fever plus convulsion was present in $70.6 \%$, followed by coma $41.2 \%$. The death rate was $6 \%$, and $64.7 \%$ had neurological sequelae.Conclusion: Acute encephalitis is an important cause of neurological sequelae in children because of the difficulties of diagnosis and management. Key words: Acute encephalitis, Public health, Child, Sequelae.

\section{INTRODUCTION}

Une encéphalite est caractérisée par un processus aigu et inflammatoire qui affecte le parenchyme du système nerveux central et qui est associé à des signes de dysfonction cérébrale [1]. L'étiologie des encéphalites reste inconnue dans la majorité des cas (plus de 50\%), mais reste dominée par les causes infectieuses, et parmi elles, les virus occupent une place prépondérante. Elle est grave car pourvoyeuse de séquelles neuropsychiatriques, d'invalidités et voir même mortelle parfois [2,3]. Le diagnostic est suspecté devant l'association de signes neurologiques centraux (convulsions, signes déficitaires où troubles de conscience), d'une méningite à liquide clair (le plus souvent lymphocytaire) et confirmé par les sérologies, la PCR et l'imagerie [4,5]. Le pronostic est fonction de la rapidité et de la qualité de la prise en charge mais aussi du terrain. Il est urgent de recherché l'étiologie de l'affection afin d'adapter le traitement spécifique, de prévenir l'évolution vers les complications voir les séquelles; mais aussi de mieux gérer l'usage des antibiotiques. C'est une entité complexe du fait de la difficulté diagnostique et de traitement spécifique adapté. [5,6]. Au Mali, la problématique de prise en charge et l'absence d'étude sur l'encéphalite chez l'enfant nous a conduits à faire cette étude.

\section{Patients et Méthode}

Notre étude s'est déroulée à Bamako (Mali), au département de pédiatrie du Centre HospitaloUniversitaire Gabriel Touré. Il s'agit d'une étude rétrospective. Elle s'est déroulée de Janvier 2015 à Décembre 2015 soit une période de 12 mois. Les 
dossiers des enfants de 0 à 15 ans hospitalisés dans le service de pédiatrie pour encéphalites aiguës étaient inclus. N'étaient pas inclus les dossiers dont l'âge du patient est supérieur à 15 ans au moment du diagnostic, ou admis en dehors de la période d'étude. Les paramètres étudiés pour l'évaluation neurologique étaient : la conscience, la sensibilité, la vision, le langage, l'audition et le tonus. L'existante de séquelles liée à la présence d'un ou plusieurs des signes sus cités. La guérison était définit par une récupération neurologique totale sans séquelle.

\section{Résultats}

Pendant notre période d'étude nous avons collecté 34 cas d'encéphalite sur 4727 hospitalisations dans le département de pédiatrie du CHU Gabriel Toure soit une fréquence de $0,72 \%$. La tranche d'âge de 12 à 59 mois était la plus représentée avec 47,1\% (Figure1). La prédominance a été masculine avec un sex-ratio de 1,8. La majorité des cas d'encéphalites aiguës ont été enregistrées au deuxième trimestre de l'année avec un pic en Mai soit 9 cas (26,47\%) ; (Figure 2), 38,23\% de nos patients étaient admis pour convulsion fébrile associée d'autres symptômes (Tableau I). Dans notre série $20,6 \%$ de nos enfants avaient des antécédents personnels de pathologies neurologiques, $58,9 \%$ des enfants étaient fébrile dont 32,4\% d'hyperthermie. A l'examen d'entrée $50 \%$ des patients présentaient une convulsion, suivie de coma $(41,2 \%)$ et les autres signes d'atteinte neuro-méningé. La Tomodensitométrie a été réalisée chez 9 patients et elle était anormale dans $89 \%$ des cas, 7 de nos patients avaient bénéficiés l'EEG (électroencéphalogramme) et il était anormal dans $71 \%$ des cas, la ponction lombaire était faite chez 28 patients et elle est revenue clair dans $85,7 \%$ des cas. Dans notre série $70,6 \%$ des enfants ont consultés 10 jours après le début des symptômes, $64,7 \%$ des patients sont guéris avec des séquelles neurologiques (tableau II). La majorité de nos patients ont eu comme prise en charge une antibiothérapie, un anticonvulsivant, la corticothérapie et la kinésithérapie dans 76,47\% des cas (Tableau III). Dans notre série les principales séquelles étaient le trouble du tonus $(14,7 \%)$, l'hémiplégie $(14,7 \%)$. Nous déplorons 2 décès soit $(5,9 \%)$ des cas.

\section{DISCUSSION}

Malgré, les difficultés liées aux biais de remplissage de certains dossiers de patients, nous avons pu collecter des données qui ont aboutis à des résultats. Au cours de l'année 2015 nous avons enregistré 4727 enfants dans le département de pédiatrie dont 34 cas d'encéphalites aigües, soit une fréquence hospitalière de $0,72 \%$. L'encéphalite survient à tout âge chez l'enfant. Nous avons retrouvé une fréquence plus élevée dans la tranche d'âge de 12 à 59 mois soit 47,1\%. Ce résultat est similaire à celui d'Aurelius $\mathrm{P}$ et al qui ont eu une prévalence élevée chez les enfants de moins de 10 ans avec un pic autour de 5 ans [5], de même que l'étude de R. Sonneville et al qui avaient eu une prévalence élevée chez les enfants de moins de 10 ans avec maximum autour de 5 à 8 ans dans 38,2\% des cas [3]. Ces résultats sont différents de l'étude américaine dont le maximum de fréquence concernait les nourrissons de moins de 1 an dans $51,3 \%$ [7]. A cet âge l'enfant est en pleine croissance avec des structures cérébrales vulnérables et est exposé à des difficultés scolaires et d'intégration sociale. Le CHU Gabriel Touré la seule structure du pays de troisième niveau qui dispose d'un département de pédiatrie. Cette structure reçoit de ce fait tous les patients en urgence vitale. La prédominance masculine dans notre étude a été retrouvée dans l'étude d'Aurelius $\mathrm{P}$ et al: 1,7 [7]. Dans notre série 58,9\% des patients avaient de la fièvre à l'admission, beaucoup d'auteurs ont rapportés la fièvre comme signe de début de l'encéphalite [1;9]. La majorité de nos patients transitent par de structures sanitaires et par conséquent reçoivent des antipyrétiques avant d'être référer à l'hôpital. Les signes cliniques à l'examen étaient dominés par la convulsion (50\%), le coma $(41,2 \%)$ dans notre étude, nos résultats sont similaires à celui de Whytley RJ $[9,10]$ et de celui de Glaser CA et al [6], par contre Kennedy PG à retrouvé un chiffre supérieur pour la convulsion $(63 \%)$ et un taux inferieur pour le coma $(30 \%)$. $[1,11]$. Les signes de l'encéphalite ne sont pas spécifiques, la fièvre est constante, associée à un ou plusieurs des signes suivants (troubles de la conscience, crises convulsives, signes de focalisation, troubles du comportement, troubles neurovégétatifs) Kennedy PG. [1,10]. L'aspect du LCR était clair dans $85,7 \%$ des cas dans notre étude qui est supérieur à l'étude Française avec environ $80 \%$ de LCR clair [8, 10]. Nous déplorons l'absence d'investigations complémentaires (Fond d'œil, l'EEG et la TDM) chez plus de la moitié de nos patients par faute de moyen financier, 1 étude d'E. Bissagnene et $\mathrm{K}$. Domoua est inferieur à nous avec $50 \%$ d'examens complémentaires non réalisés pour faute de moyen financier [7]. La difficulté diagnostique de l'encéphalite et la forte suspicion de méningite bactérienne au début pourrait expliquer le fort taux d'utilisation d'antibiotique chez nos patients $(76,47 \%)$. Nous regrettons un fort taux de séquelles neurologiques dans notre série $(64,7 \%)$ dominés par les troubles de tonus et l'hémiplégie (respectivement $14,7 \%$ chacun), deux 
cas décès $(5,9 \%)$. Cela s'explique sûrement par le retard de prise en charge par faute de moyen financier $(70,6 \%$ de nos patients ont été prise en charge après 10 jours d'évolution de la maladie). Nos donnés sont proches de celui d'E. Bissagnene et E. Damoua [7].

\section{CONCLUSION}

L'encéphalite de l'enfant bien que rare $(0,72 \%)$ est grave à cause des séquelles $(64,7 \%)$, qui constituent un véritable problème d'insertion sociale. Les signes cliniques à l'examen étaient dominés par la convulsion $(50 \%)$, le coma $(41,2 \%)$. La tranche d'âge de 12 à 59 mois était la plus touchée $(47,1 \%)$. La réalisation d'une étude analytique permettra une meilleure connaissance sur l'efficacité des moyens de prise en charge et les étiologies.

\section{Conflit d'intérêt : Aucun}

\section{Références}

1. Kennedy PG. Viral encephalitis. J Neurol 2011; 252: 268-72. [Medline]

2. Whitley RJ, Gnann JW. Viral encephalitis: Familiar infections and emerging pathogens. Lancet 2012; 359:507-13. [Medline]

3. Whitley RJ, Kimberlin DW. Viral encephalitis. Pediatr Rev 2013; 20:

192-8.

4. Eelco FW. Acute viral encephalitis. Clinical Practice of Critical Care Neurology. 2011; 252-61.
5. Steiner I, Budka H, Chaudhuri A, et al. Viral encephalitis: A review of diagnostic methods and guidelines for management. Eur J Neurol 2011; 12:331-43. [Medline]

6. Thwaites GE, Tran TH. Tuberculosis meningitis: many questions, too few answers. Lancet Neurol 2009; 4: 160-70.

7. Aurelius E, Johansson B, Skoldenberg B, StalandA, Forsgren M. Rapid diagnosis of herpes simplex encephalitis by nested polymerase chain reaction assay of cerebrospinal fluid. Lancet 2012; 337:189-92.

8. R. Sonneville, M. Wolff. Encéphalomyélite aiguë disséminée et encéphalites post-infectieuses graves- Acute disseminated encephalomyelitis and severe post-infectious encephalitis. Réanimation (2010) 16, 452-462

9. Whitley RJ. Viral encephalitis. N Engl J Med 2013; 323: 242-50.

10. Cambonie G, Houdon L, Rivier F, Bongrand AF, Echenne B. Infantile bilateral striatal necrosis following measles. Brain Dev 2010;22:221-3.

11. Sehulster L, Chinn RY. Guidelines for environmental infection control in health-care facilities. Recommendations of $\mathrm{CDC}$ and the Healthcare Infection Control Practices Advisory Committee (HICPAC). MMWR Recomm Rep 2013; 52 (RR-10): 1-42.

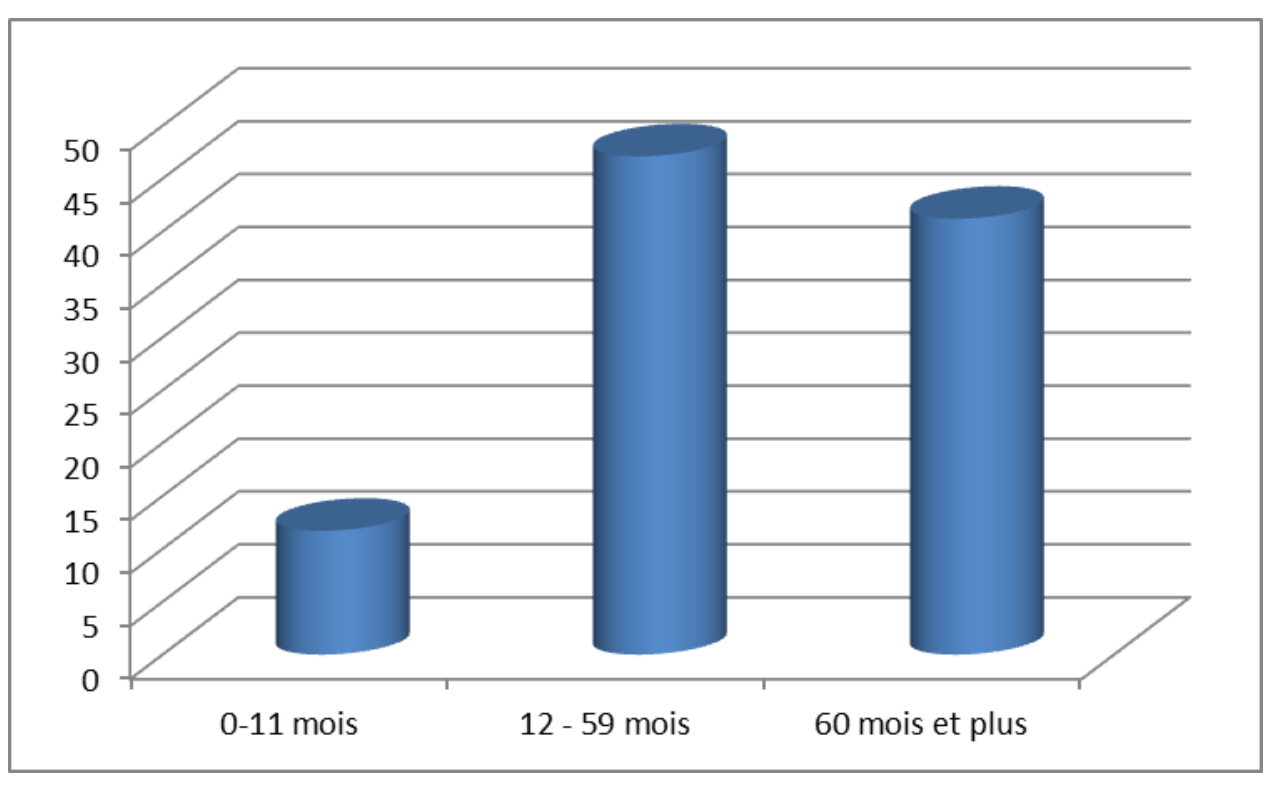

Figure I : Patients selon la tranche d'âge 


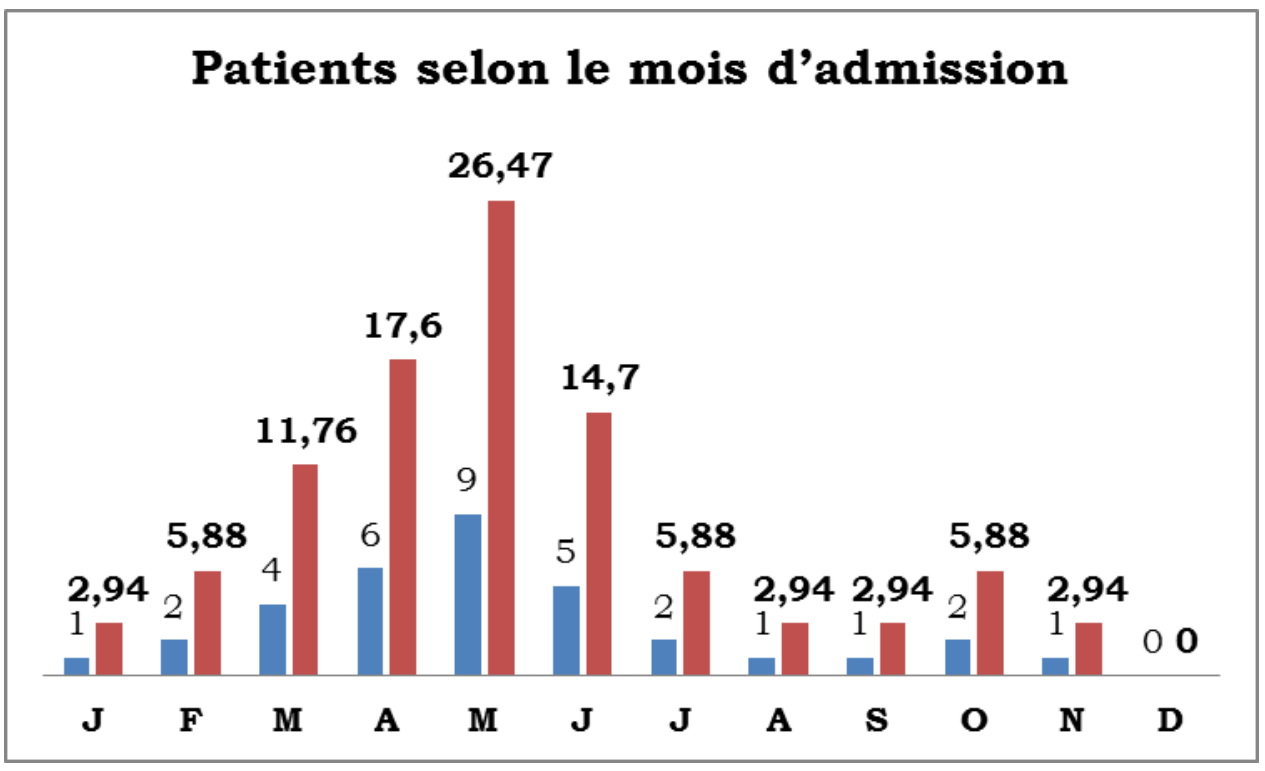

Figure II : Patients selon le mois d'admission

Tableau I : Patients selon le motif de consultation

\begin{tabular}{lcc}
\hline Motif de consultation & Effectif & Pourcentage \\
\hline Convulsion fébrile & 13 & 38,23 \\
Autres symptômes plus la convulsion fébrile & $\mathbf{2 1}$ & $\mathbf{6 1 , 7 7}$ \\
\hline Total & $\mathbf{3 4}$ & $\mathbf{1 0 0}$ \\
\hline
\end{tabular}

Tableau II : Patients selon le l'évolution

\begin{tabular}{lcc}
\hline Devenir & Effectif & Pourcentage \\
\hline Guérison sans séquelle & 10 & 29,4 \\
Guérison avec séquelles & $\mathbf{2 2}$ & $\mathbf{6 4 , 7}$ \\
Décédés & 2 & 5,9 \\
\hline Total & $\mathbf{3 4}$ & $\mathbf{1 0 0}$ \\
\hline
\end{tabular}

Tableau III : Patients selon le traitement reçu à l'admission 


\begin{tabular}{lcc}
\hline Traitement reçu à l'admission & Effectif & Pourcentage \\
\hline Antibiotique, anticonvulsivant, corticoïde, kinésithérapie, & $\mathbf{2 6}$ & $\mathbf{7 6 , 4 7}$ \\
antipyrétique & & \\
Antibiotique, antipyrétique, corticoïde & 3 & 8,82 \\
Anticonvulsivant, kinésithérapie & 2 & 5,88 \\
Anticonvulsivant & 2 & 5,88 \\
Kinésithérapie & 1 & 2,94 \\
\hline Total & $\mathbf{3 4}$ & $\mathbf{1 0 0}$ \\
\hline
\end{tabular}

